\title{
The large charge limit of scalar field theories, and the Wilson-Fisher fixed point at $\epsilon=0$
}

\author{
G. Arias-Tamargo ${ }^{a, b}$ D. Rodriguez-Gomez ${ }^{a, b}$ and J.G. Russo ${ }^{c, d}$ \\ ${ }^{a}$ Department of Physics, Universidad de Oviedo, \\ C/ Federico García Lorca 18, 33007 Oviedo, Spain \\ ${ }^{b}$ Instituto Universitario de Ciencias y Tecnologías Espaciales de Asturias (ICTEA), \\ C/ de la Independencia 13, 33004 Oviedo, Spain \\ ${ }^{c}$ Institució Catalana de Recerca i Estudis Avançats (ICREA), \\ Pg. Lluis Companys, 23, 08010 Barcelona, Spain \\ ${ }^{d}$ Departament de Física Cuántica i Astrofísica and Institut de Ciències del Cosmos, \\ Universitat de Barcelona, Martí Franquès, 1, 08028 Barcelona, Spain \\ E-mail: guillermo.arias.tam@gmail.com, d.rodriguez.gomez@uniovi.es, \\ jorge.russo@icrea.cat
}

ABSTRACT: We study the sector of large charge operators $\phi^{n}$ ( $\phi$ being the complexified scalar field) in the $O(2)$ Wilson-Fisher fixed point in $4-\epsilon$ dimensions that emerges when the coupling takes the critical value $g \sim \epsilon$. We show that, in the limit $g \rightarrow 0$, when the theory naively approaches the gaussian fixed point, the sector of operators with $n \rightarrow \infty$ at fixed $g n^{2} \equiv \lambda$ remains non-trivial. Surprisingly, one can compute the exact 2-point function and thereby the non-trivial anomalous dimension of the operator $\phi^{n}$ by a full resummation of Feynman diagrams. The same result can be reproduced from a saddle point approximation to the path integral, which partly explains the existence of the limit. Finally, we extend these results to the three-dimensional $O(2)$-symmetric theory with $(\bar{\phi} \phi)^{3}$ potential.

Keywords: Conformal Field Theory, Effective Field Theories, Renormalization Group

ARXIV EPRINT: 1908.11347 


\section{Contents}

1 Introduction and summary 1

2 The Wilson-Fisher fixed point for a complex scalar field 3

3 The double scaling limit on correlation functions 4

3.1 Diagrammatic computation 4

$\begin{array}{lll}3.2 & \text { Saddle-point derivation } & 7\end{array}$

4 The double-scaling limit in $d=3 \quad 8$

$\begin{array}{ll}\text { A Real space renormalization } & 10\end{array}$

\section{Introduction and summary}

Despite decades of huge research efforts, Quantum Field Theory (QFT) is far from analytic reach beyond perturbative approaches which, in practice, typically comprise the computation of certain observables to a few loop accuracy. It thus comes as a very welcome surprise that, in certain cases, it is possible to identify special limits which lead to drastic simplifications and sometimes to a reorganization of perturbation theory. A prototypical example is the large $N$ limit discovered by 't Hooft. In a gauge theory, one takes the rank $N$ of the gauge algebra to infinity at the same time that the Yang-Mills coupling $g_{\mathrm{YM}}$ is sent to zero in such a way that the 't Hooft coupling $g_{\mathrm{YM}}^{2} N$ is fixed. This limit selects planar diagrams in the perturbative expansion of the theory, which naturally organize themselves into a genus expansion very reminiscent of a string theory, a connection that has been intensively studied over the last two decades.

A different approach is to explore asymptotic regimes in the space of operators in a certain QFT, in particular focusing on those with large charge $n$ under a global symmetry of the theory. This remarkable suggestion was made in [1] and it was further explored in many relevant papers including [2-15]. A new perturbation expansion emerges in terms of a small effective coupling represented by the inverse of the charge, $1 / n$ (see also $[16,17]$ for other interesting physical applications).

Very recently, a new "double-scaling" large charge limit was introduced in [18] in the context of $\mathcal{N}=24 \mathrm{~d}$ superconformal field theories. In this case, supersymmetric localization provides an efficient method to compute "extremal" correlators of chiral primary operators $\left(\operatorname{Tr} \phi^{2}\right)^{n}$ [19] (being $\phi$ the scalar field in the vector multiplet). In the $\mathcal{N}=2$ SCFT context, the double scaling limit of [18] corresponds to taking $g_{\mathrm{YM}} \rightarrow 0, n \rightarrow \infty$ keeping $\lambda=g_{\mathrm{YM}}^{2} n$ fixed. This limit systematically isolates, at each loop order in the perturbative expansion of SQCD, a certain contribution. Its existence requires that at $k$ loops, any extremal 
correlator has a leading behavior $n^{k}$, which remarkably turns out to be the case to all loop orders. Detailed aspects of this limit were discussed in the relevant papers [20, 21]. It was recently understood in an important paper [22] that this limit can be viewed as the standard 't Hooft limit of an associated random matrix model. In particular, this explains why the limit exists, at least in this theory. In addition, the matrix model interpretation of [22] allows one to obtain the exact $\lambda$ dependence in correlators in closed form by employing standard matrix model techniques.

An obvious question is whether the existence of the double-scaling, large charge limit is a peculiarity of highly supersymmetric theories such as $\mathcal{N}=2$ SCFTs. In this note we find that an analogous limit exists for a familiar non-supersymmetric theory, namely scalar field theory with quartic potential. We will show that the very familiar Wilson-Fisher (WF) fixed point for the $O(2)$ theory provides perhaps the simplest example where one can study non-trivial correlation functions in said limit, by means of a complete resummation of Feynman diagrams. ${ }^{1}$

One may more generally consider the $O(N)$ model in $4-\epsilon$ dimensions, but for simplicity we shall restrict the discussion to the $N=2$ case. This can be recast as the theory for a complex scalar $\phi$ with a quartic interaction controlled by a coupling $g$. Appropriately tuning the mass parameter, there is a renormalization group flow to the Wilson-Fisher fixed point where $g \sim \epsilon$. One of the remarkable applications of the $\epsilon$ expansion is to extrapolate the results to $\epsilon=1$, where the model describes the ferromagnetic transition of the $3 \mathrm{~d}$ Ising model. Although this limit is far from the perturbative regime, the analytical results nevertheless remarkably agree with the numerical values for various critical exponents. On the other hand, in taking the limit $\epsilon \rightarrow 0$ the theory is simply led to the gaussian fixed point in $d=4$. However, the limit of $[18,20-22]$ suggests that one can consider sectors of large global charge which might have non-trivial dynamics. Specifically, we consider operators $\mathcal{O}_{n} \equiv \phi^{n}$ of $\mathrm{U}(1)$ charge $n$ and engineering dimension $n\left(1-\frac{\epsilon}{2}\right)$. It turns out that in the limit $g \rightarrow 0$, the sector of operators with $n \rightarrow \infty$ such that $\lambda=g n^{2}$ is fixed, have nontrivial correlators, which can be exactly computed through a resummation of the surviving Feynman diagrams. We also provide an alternative derivation from the path integral: in the double scaling limit, it is dominated by a saddle-point, giving rise to the same correlation function previously obtained diagrammatically. The saddle-point calculation suggests that a similar limit may exist in other theories. In particular, we also consider the $O(2)$ theory in three dimensions for a potential $(\bar{\phi} \phi)^{3}$, where we identify the relevant limit and compute the exact two-point correlation function for the operators $\phi^{n}, \bar{\phi}^{n}$.

Let us comment on some interesting open problems. It would be interesting to consider higher point functions in detail. A preliminary observation is as follows. Consider, for instance, a 3-point function $\left\langle\mathcal{O}_{n}(x) \mathcal{O}_{n}(y) \mathcal{O}_{2 n}^{-}(0)\right\rangle$ in the simplest context of the $O(2)$ model studied in this note. One can show that, to next-to-leading order, there are diagrams surviving the limit, yielding a result consistent with the structure dictated by conformal symmetry. Clearly, it would be of interest to extend this study to all orders and to arbitrary

\footnotetext{
${ }^{1}$ The question on the large charge limit of the WF fixed point was also raised in [22], which appeared as this paper was being completed.
} 
$k$-point functions. It would also be very interesting to systematically study the structure of 2-point functions following [7]. This might lead to universal relations involving the central charges of the conformal algebra. A challenging problem is to see if, as suggested in [22], the double scaling limit of the $O(2)$ theory can be understood as a 't Hooft limit of a "dual" random matrix model. One may also study large $\mathrm{R}$ charge correlators in ABJM theory in the same limit, which could be compared against results from supersymmetric localization.

\section{The Wilson-Fisher fixed point for a complex scalar field}

Let us consider the $O(N)$ model in $d=4-\epsilon$ dimensions. This model is both of pedagogical interest -as the historic laboratory for QFT and RG- as well as of practical interest: for different values of $N$ it is known to describe various phase transitions of relevant physical systems (for instance, for $N=1$, at $\epsilon=1$, it describes the 3 d ferromagnetic transition. See e.g. [24] for an introduction). The action reads

$$
S=\int d^{4-\epsilon} x\left(\frac{1}{2}(\partial \vec{\varphi})^{2}-\frac{1}{2} m^{2} \vec{\varphi}^{2}-\frac{g}{16}\left(\vec{\varphi}^{2}\right)^{2}\right)
$$

where $\vec{\varphi}$ is the $N$ component field rotated by the $O(N)$ symmetry. As it is well-known (see e.g. [25]), upon tuning the mass to zero this flows to the Wilson-Fisher fixed point at the critical value

$$
g_{\mathrm{WF}}=\frac{32 \pi^{2}}{N+8} \epsilon .
$$

We will be interested in theories with a global U(1) charge, for which the simplest example is $N=2$. In that case the theory can be re-written as the theory for a complex scalar field in $4-\epsilon$ dimensions with action

$$
S=\int d^{4-\epsilon} x\left(\partial \bar{\phi} \partial \phi-m^{2} \bar{\phi} \phi-\frac{g}{4}(\bar{\phi} \phi)^{2}\right)
$$

With these conventions, the Feynman rule for the vertex is just $-i g$. We will be interested in the critical case where $m^{2}=0$.

Note that this construction allows one to take the $g \rightarrow 0$ limit along a family of Conformal Field Theories. Nevertheless, since we are ultimately interested in the extreme weak coupling limit, we may alternatively simply consider the $g(\bar{\phi} \phi)^{2}$ theory in $d=4$.

It is easy to compute the anomalous dimension of scalar operators of the form $\mathcal{O}_{n}=\phi^{n}$ to $O(g)$. One finds $\gamma_{\mathcal{O}_{n}} \sim g n^{2} \sim \epsilon n^{2}$ (see e.g. [26]). The emergence of the combination $\lambda=g n^{2} \sim \epsilon n^{2}$ suggests the existence of a double scaling limit:

$$
g \rightarrow 0, \quad n \rightarrow \infty, \quad \lambda=g n^{2} \text { fixed }
$$

The existence of the limit may also be suggested by earlier investigations on the exponentiation property of multiparticle amplitudes [27-29]. 


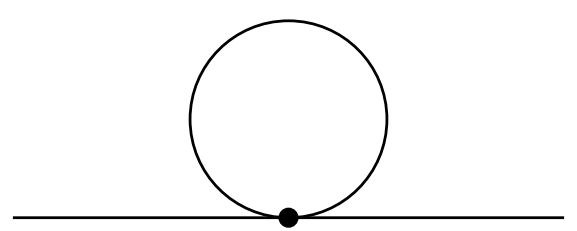

Figure 1. Bubble diagram.

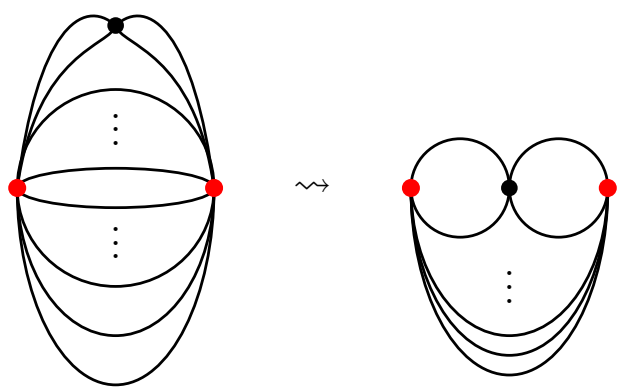

Figure 2. The relevant one-loop diagram and its Kermit the frog representation.

\section{The double scaling limit on correlation functions}

We will now investigate the limit (2.4) in the exact two-point correlation function $\left\langle\mathcal{O}_{n}(x) \overline{\mathcal{O}}_{n^{\prime}}(0)\right\rangle$, with $\mathcal{O}_{n}=\phi^{n}, \overline{\mathcal{O}}_{n}=\bar{\phi}^{n}$ in $d=4-\epsilon$ dimensions. These operators have a definite $\mathrm{U}(1)$ charge $n$ and hence they are automatically orthogonal for different charges. ${ }^{2}$

\subsection{Diagrammatic computation}

Let us first compute the 2-point functions by evaluation of the relevant Feynman diagrams. As a preliminary step, let us consider the bubble diagram in figure 1, which is ubiquitous in the perturbative expansion of such correlators.

This diagram has no dependence on the external momenta; therefore it can only be proportional to the mass. Since we will be interested in the critical theory, this diagram vanishes. Thus, when computing $\mathcal{O}_{n}$ correlators, we shall only consider diagrams that do not contain any bubble.

Let us now consider the systematics of the perturbative expansion of the correlation function $\left\langle\mathcal{O}_{n}(x) \overline{\mathcal{O}}_{n}(0)\right\rangle$. As usual, at each order in the perturbative expansion in $g$ there are several topologically different diagrams, each one coming with a certain dependence on $n$. We are going to be interested in taking $n$ to infinity, and inspection of all topologies shows that in this limit a class of them dominates over the rest. As shown below, the dominant topology can be viewed as an iteration of the one-loop diagram of figure 2, that we will call Kermit the frog's diagram [30].

At order $g^{2}$, we have the four diagrams of figure 3 above. The key point to identify the dominant diagram at large $n$, at any given loop order, is the $n$ dependence, which comes

\footnotetext{
${ }^{2}$ It should be noted that, at fixed charge $n$, the most general operators are of the form $\mathcal{O}_{n, k}=(\bar{\phi} \phi)^{k} \mathcal{O}_{n}$. We will restrict to the lowest tower with $k=0$.
} 

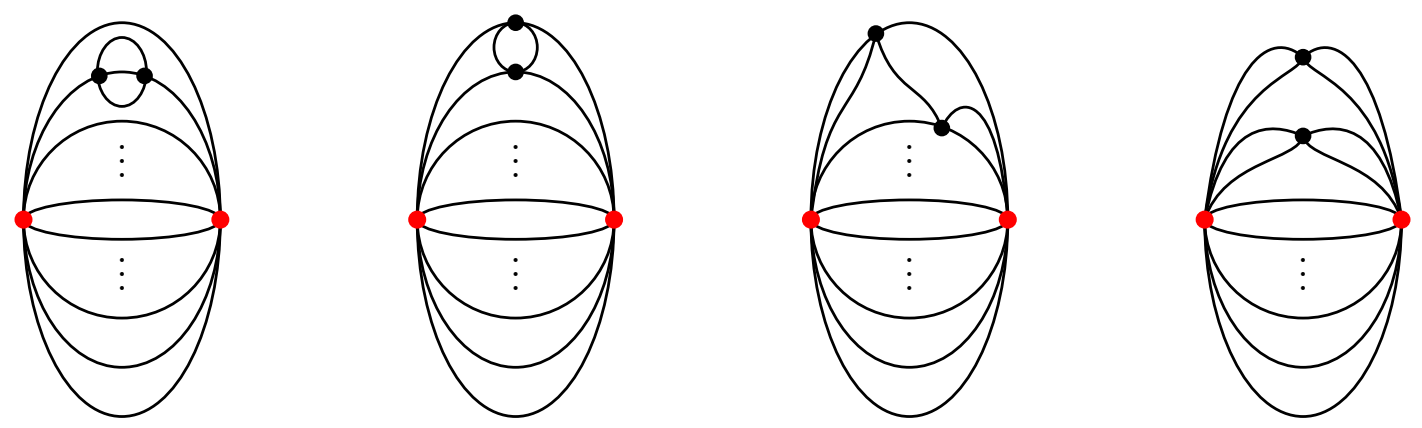

Figure 3. Four topologies contributing at order $O\left(g^{2}\right)$.

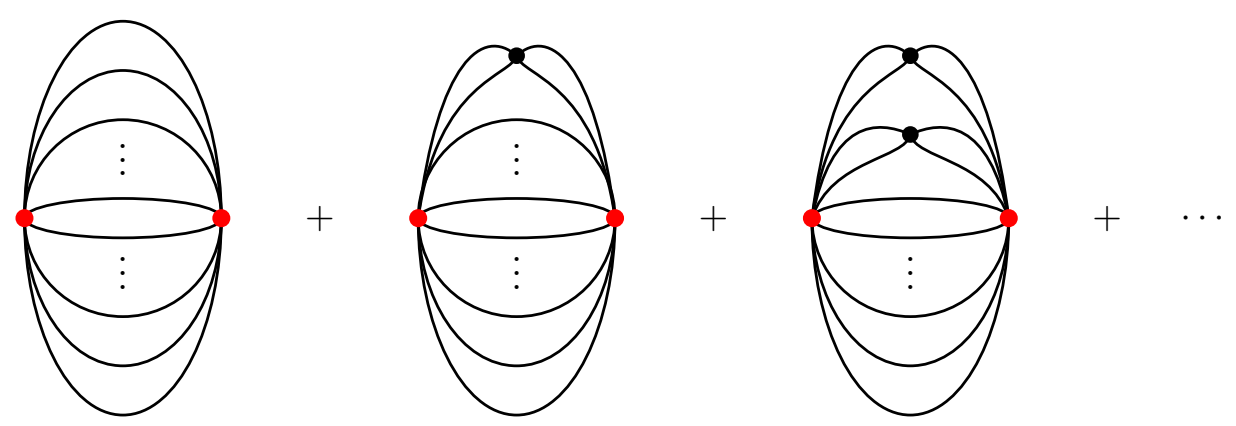

Figure 4. Diagrams contributing to $\left\langle\mathcal{O}_{n}(x) \overline{\mathcal{O}}_{n}(x)\right\rangle$ at large $n$.

from the combinatorial factor. This is given by $n ! n ! / k !$, where $k$ is the number of lines that do not undergo interactions. Therefore, the diagram that has the highest power of $n$ is the one with the smallest $k$. Using this formula, we thus find that the combinatorial factor of the fourth diagram in figure 3 is $n ! n(n-1)(n-2)(n-3)$. On the other hand, the formula $n ! n ! / k !$ implies that the combinatorial factors of the first, second and third diagrams are $n ! n, n ! n(n-1)$ and $n ! n(n-1)(n-2)$ respectively (we omit numerical coefficients standing from symmetrization, which do not affect the $n$ dependence). Thus, for large $n$, the diagram on the right dominates. We will call this one the two-loop Kermit diagram.

Now consider the general $m$-loop diagram with $m$ vertices. The lines in each vertex can go either to another vertex or join some of the $n$ lines of the operators $\phi^{n}$ or $\bar{\phi}^{n}$. The diagram which has a smaller number of lines that do not undergo interactions is when two lines of each vertex join two of the $n$ lines of the operator $\phi^{n}$ and the other two lines join two of the $n$ lines of the operator $\bar{\phi}^{n}$ (it is not possible to have three lines of the vertex joining three lines of the operators $\phi^{n}$ because of charge conservation; vertices are of the form $\phi \phi \bar{\phi} \bar{\phi})$. This corresponds to the iteration of the Kermit diagram and has a combinatorial factor $n !^{2} /(n-2 m)$ ! which has the highest power of $n$ (see (3.1) and below for the derivation of the combinatorial factor at $m$ loop order including other symmetry factors).

Thus, we conclude that a class of diagrams dominate the correlation function, the $m$-loop Kermit diagrams of figure 4 . Denoting by $K_{m}$ the contribution from the Kermit diagram with $m$ interaction vertices, the correlator is

$$
\left\langle\mathcal{O}_{n}(x) \overline{\mathcal{O}}_{n}(0)\right\rangle=n ! \sum_{m=0}(-i g)^{m} K_{m} \frac{1}{4^{m}} \frac{n !}{(n-2 m) !} \frac{1}{m !} .
$$


The combinatorial factor can be understood from figure 4 . There are $n$ lines on each side, giving rise to a factor $(n !)^{2}$ obtained by permutations. Then one must divide over the number of permutations that lead to equivalent configurations. There is a factor $1 /(n-2 m)$ ! associated with the permutations of the $n-2 m$ lines that do not undergo interaction. There is also a factor $1 / 2^{m}$ on each side associated with the permutations of the pair of lines in the $m$ loops. The factor $1 / m$ ! originates from the expansion of the exponential of the interaction term.

Using the de Moivre-Stirling formula, for $n \gg 1$ one obtains

$$
\frac{n !}{(n-2 m) !} \approx n^{2 m}, \quad n \gg 1 \text {. }
$$

Therefore we can define the limit

$$
n \rightarrow \infty, \quad g \rightarrow 0, \quad \lambda=g n^{2}=\text { fixed } .
$$

The correlator then becomes

$$
\left\langle\mathcal{O}_{n}(x) \overline{\mathcal{O}}_{n}(0)\right\rangle=n ! \sum_{m=0} K_{m}\left(\frac{-i \lambda}{4}\right)^{m} \frac{1}{m !} .
$$

To further proceed, note that, in position space, the Kermit diagram $K_{m}$ is

$$
\begin{aligned}
K_{m} & =G(0, x)^{n-2 m} \prod_{i=1}^{m} \int d^{4} z_{i} G\left(0, z_{i}\right)^{2} G\left(z_{i}, x\right)^{2} \\
& =G(0, x)^{n}\left(\frac{1}{G(0, x)^{2}} \int d^{4} z G(0, z)^{2} G(z, x)^{2}\right)^{m},
\end{aligned}
$$

where $G(x, y)$ is the propagator of the $\phi$ field. Thus

$$
\left\langle\mathcal{O}_{n}(x) \overline{\mathcal{O}}_{n}(0)\right\rangle=n ! G(0, x)^{n} \sum_{m=0}\left(\frac{-i \lambda \mathcal{K}}{4}\right)^{m} \frac{1}{m !},
$$

with

$$
\mathcal{K}=\frac{1}{G(0, x)^{2}} \int d^{4} z G(0, z)^{2} G(z, x)^{2} .
$$

Since $n ! G(0, x)^{n}=\left\langle\mathcal{O}_{n}(x) \mathcal{O}_{n}(0)\right\rangle_{0}$ is the correlation function in the free theory, and the sum can be trivially resumed, we find

$$
\left\langle\mathcal{O}_{n}(x) \overline{\mathcal{O}}_{n}(0)\right\rangle=\left\langle\mathcal{O}_{n}(x) \overline{\mathcal{O}}_{n}(0)\right\rangle_{0} e^{-i \frac{\lambda \mathcal{K}}{4}} .
$$

Next, consider the computation of $\mathcal{K}$, which is carried out in the appendix. Note that $\mathcal{K}$ represents the $O(g)$ correction to the $\mathcal{O}_{2}$ correlator. We have

$$
\mathcal{K}=-\frac{i}{8 \pi^{2}} \log \left(\Lambda^{2} x^{2}\right) .
$$

As a cross-check of this result, one can see that in the $N=1$ case, and upon appropriately taking into account numerical conventions, this yields the correct $O(g)$ anomalous dimension of the $\mathcal{O}_{n}$ operator (cf. for example [26]). 
Thus

$$
\left\langle\mathcal{O}_{n}(x) \overline{\mathcal{O}}_{n}(0)\right\rangle=\left\langle\mathcal{O}_{n}(x) \overline{\mathcal{O}}_{n}(0)\right\rangle_{0} \frac{1}{|x|^{\frac{\lambda}{16 \pi^{2}}}}
$$

Since in position space

$$
G(0, x)=\frac{1}{4 \pi^{2}} \frac{1}{|x|^{2}},
$$

we finally find

$$
\left\langle\mathcal{O}_{n}(x) \overline{\mathcal{O}}_{n}(0)\right\rangle=\frac{n !}{\left(4 \pi^{2}\right)^{n}|x|^{2\left(n+\frac{\lambda}{32 \pi^{2}}\right)}} .
$$

In particular, this gives the following formula for the dimension of the $\mathcal{O}_{n}$ operator in the double scaling limit

$$
\Delta_{\mathcal{O}_{n}}=n+\frac{\lambda}{32 \pi^{2}}
$$

\subsection{Saddle-point derivation}

The underlying reason behind the existence of a large charge limit can be understood from a saddle-point calculation. It is convenient to rescale the scalar field and define new variables

$$
\sigma=g^{\frac{1}{4}} \phi, \quad \bar{\sigma}=g^{\frac{1}{4}} \bar{\phi} .
$$

The correlator is then given by

$$
\left\langle\mathcal{O}_{n}\left(x_{1}\right) \overline{\mathcal{O}}_{n}\left(x_{2}\right)\right\rangle=\frac{1}{g^{\frac{n}{2}} Z} \int D \sigma D \bar{\sigma} e^{-S},
$$

where the Euclidean action, including source terms, is given by

$$
S=\int d^{4} x\left(g^{-\frac{1}{2}} \partial \bar{\sigma} \partial \sigma+\frac{1}{4}(\bar{\sigma} \sigma)^{2}-n \delta\left(x-x_{1}\right) \log \sigma-n \delta\left(x-x_{2}\right) \log \bar{\sigma}\right) .
$$

In the large $n$ limit, this integral is dominated by a saddle-point. Indeed, the saddlepoint analysis is very similar to the one carried out in section 2.3 of [7]. The saddle-point equations are given by

$$
\partial^{2} \sigma=-n g^{\frac{1}{2}} \delta\left(x-x_{2}\right) \frac{1}{\bar{\sigma}}+\frac{1}{2} g^{\frac{1}{2}} \bar{\sigma} \sigma^{2}, \quad \partial^{2} \bar{\sigma}=-n g^{\frac{1}{2}} \delta\left(x-x_{1}\right) \frac{1}{\sigma}+\frac{1}{2} g^{\frac{1}{2}} \bar{\sigma}^{2} \sigma .
$$

The crucial point is that, in the limit $g \rightarrow 0, n \rightarrow \infty$, where $\lambda=n^{2} g=$ fixed, the interaction term can be ignored. The resulting equations become

$$
\bar{\sigma} \partial^{2} \sigma=-\lambda^{\frac{1}{2}} \delta\left(x-x_{2}\right), \quad \sigma \partial^{2} \bar{\sigma}=-\lambda^{\frac{1}{2}} \delta\left(x-x_{1}\right) .
$$

These equations are now equivalent to those discussed in [7]. The solution is given by

$$
\sigma(x)=\lambda^{1 / 4} \frac{e^{i \beta_{0}}\left|x_{1}-x_{2}\right|}{2 \pi\left(x-x_{2}\right)^{2}}, \quad \bar{\sigma}(x)=\lambda^{1 / 4} \frac{e^{-i \beta_{0}}\left|x_{1}-x_{2}\right|}{2 \pi\left(x-x_{1}\right)^{2}} .
$$

Let us now substitute this solution into the action. Consider first the interaction term. This is absent in [7] and it is indeed the interesting part in our case. We have

$$
\int d^{4} x \frac{1}{4}(\bar{\sigma} \sigma)^{2}=\frac{\lambda}{4(2 \pi)^{4}} \int d^{4} x \frac{\left|x_{1}-x_{2}\right|^{4}}{\left(x-x_{1}\right)^{4}\left(x-x_{2}\right)^{4}} .
$$


The integral can be computed by using (A.3), (A.5), upon shifting $x \rightarrow x+x_{1}$. We get

$$
\int d^{4} x \frac{1}{x^{4}\left(x-\left(x_{2}-x_{1}\right)\right)^{4}}=\frac{4 \pi^{2}}{\left(x_{2}-x_{1}\right)^{4}} \log \left|x_{2}-x_{1}\right|
$$

Thus

$$
\int d^{4} x \frac{1}{4}(\bar{\sigma} \sigma)^{2}=\frac{\lambda}{32 \pi^{2}} \log \left(x_{1}-x_{2}\right)^{2} .
$$

Let us now consider the remaining terms in the action. Following [7], we have

$$
\begin{aligned}
g^{-\frac{1}{2}} \int d^{4} x \partial \bar{\sigma} \partial \sigma-n \log \left(\sigma\left(x_{1}\right) \bar{\sigma}\left(x_{2}\right)\right) & =-n \log \left(\sigma\left(x_{1}\right) \bar{\sigma}\left(x_{2}\right)\right)+n \\
& =-\frac{n}{2} \log \lambda+n+n \log \left(2 \pi\left(x_{1}-x_{2}\right)\right)^{2}
\end{aligned}
$$

Putting all pieces together, we find

$$
\left\langle\mathcal{O}_{n}(x) \overline{\mathcal{O}}_{n}(0)\right\rangle=\frac{n !}{\left(4 \pi^{2}\right)^{n}\left|x_{1}-x_{2}\right|^{2\left(n+\frac{\lambda}{32 \pi^{2}}\right)}} \quad n ! \sim(2 \pi)^{1 / 2} n^{n+\frac{1}{2}} e^{-n}
$$

which is precisely the result (3.12) found from the perturbative calculation based on resumming Feynman diagrams.

It is worth noting that $(3.23)$ can also be written as

$$
\left\langle\mathcal{O}_{n}(x) \overline{\mathcal{O}}_{n}(0)\right\rangle=\frac{n !}{\left(4 \pi^{2}\right)^{n}|x|^{2 n\left(1+\frac{\hat{\lambda}}{32 \pi^{2}}\right)}}
$$

where $\widehat{\lambda}=\frac{\lambda}{n}=g n$. This makes contact with the limit of [27-29], recently discussed in $[31,32]$, where $\widehat{\lambda}$ is kept fixed. More precisely, on general grounds, correlation functions for large charge operators admit a double, 't Hooft-like, expansion in $n, \hat{\lambda}$, so that $\Delta=$ $\sum_{i=0}^{\infty} n^{1-i} F_{n}(\widehat{\lambda})$. At the same time, for weak $\widehat{\lambda}$ coupling, $F_{0}$ must admit a perturbative expansion $F_{0}=1+a \hat{\lambda}+\cdots$, where, by explicit computation, $a=\frac{1}{32 \pi^{2}}$. Fixed $\lambda$ implies $\hat{\lambda} \ll 1$ when $n \gg 1$, which corresponds to the weak coupling regime in the $\hat{\lambda}$ expansion. In this way our formula above is recovered as the $n \rightarrow \infty$ (akin to the planar limit in the familiar 't Hooft $\frac{1}{N}$ expansion) at weak $\widehat{\lambda}$ coupling. From this perspective, the $\frac{1}{n}$ corrections to the saddle-point approximation reconstruct the double expansion described above.

\section{The double-scaling limit in $d=3$}

We can similarly consider $(\bar{\phi} \phi)^{3}$ theory in $d=3$, defined by the action

$$
S=\int d^{3-\epsilon} x\left(\partial \bar{\phi} \partial \phi-m^{2} \bar{\phi} \phi-\frac{g}{3 !}(\bar{\phi} \phi)^{3}\right) .
$$

It should be noted that this model cannot describe the $\epsilon \rightarrow 1$ limit of the WF fixed point discussed in the previous section, as it contains a sextic (as opposed to quartic) interaction. Note that in both cases the interaction term is classically marginal in their respective dimensions and that the fixed points lie in the perturbative regime. In fact, just 


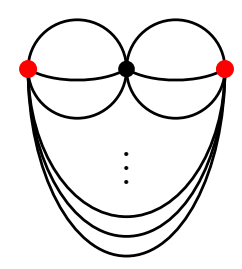

Figure 5. Sleeping Kermit. The diagram represents the leading non-trivial contribution to the two-point correlation function of the $d=3$ theory in the double-scaling limit.

as in the WF case above, our strategy in this $d=3$ model will be to consider large charge operators in the extreme weak coupling regime.

Let us consider the saddle-point calculation for the same correlator $\left\langle\mathcal{O}_{n}\left(x_{1}\right) \overline{\mathcal{O}}_{n}\left(x_{2}\right)\right\rangle$. After scaling $\sigma=g^{\frac{1}{6}} \phi, \quad \bar{\sigma}=g^{\frac{1}{6}} \bar{\phi}$. the action becomes

$$
S=\int d^{3} x\left(g^{-\frac{1}{3}} \partial \bar{\sigma} \partial \sigma+\frac{1}{3 !^{2}}(\bar{\sigma} \sigma)^{3}-n \delta\left(x-x_{1}\right) \log \sigma-n \delta\left(x-x_{2}\right) \log \bar{\sigma}\right) .
$$

A similar saddle-point analysis now leads to the equations

$$
\partial^{2} \sigma=-n g^{\frac{1}{3}} \delta\left(x-x_{2}\right) \frac{1}{\bar{\sigma}}+\frac{1}{12} g^{\frac{1}{3}} \bar{\sigma}^{2} \sigma^{3}, \quad \partial^{2} \bar{\sigma}=-n g^{\frac{1}{3}} \delta\left(x-x_{1}\right) \frac{1}{\sigma}+\frac{1}{12} g^{\frac{1}{3}} \bar{\sigma}^{3} \sigma^{2} .
$$

We now take the limit

$$
n \rightarrow \infty, \quad g \rightarrow 0, \quad \text { with } \lambda=n^{3} g=\text { fixed }
$$

As in $d=4$, the interaction term vanishes in the limit. The solutions of the saddle-point equations are obtained just like in the $d=4$ case, finding now

$$
\sigma(x)=\lambda^{1 / 6} \frac{e^{i \beta_{0}}\left|x_{1}-x_{2}\right|^{\frac{1}{2}}}{\sqrt{4 \pi}\left|x-x_{2}\right|}, \quad \bar{\sigma}(x)=\lambda^{1 / 6} \frac{e^{-i \beta_{0}}\left|x_{1}-x_{2}\right|^{\frac{1}{2}}}{\sqrt{4 \pi}\left|x-x_{1}\right|} .
$$

The anomalous dimension now comes from the contribution

$$
\int d^{3} x \frac{1}{3 !^{2}}(\bar{\sigma} \sigma)^{3}=\frac{\lambda}{3 !^{2}(4 \pi)^{3}} \int d^{3} x \frac{\left|x_{1}-x_{2}\right|^{3}}{\left|x-x_{1}\right|^{3}\left|x-x_{2}\right|^{3}} .
$$

This integral represents the Feynman diagram of figure 5, the "sleeping Kermit".

This integral can be done using the results in appendix, leading to

$$
\int d^{3} x \frac{1}{3 !^{2}}(\bar{\sigma} \sigma)^{3}=\frac{\lambda}{(24 \pi)^{2}} \log \left|x_{1}-x_{2}\right|^{2} \Lambda^{2} .
$$

The saddle-point calculation implies that, as in the $d=4$ case, this contribution exponentiates, leading to a correlator

$$
\left\langle\mathcal{O}_{n}(x) \overline{\mathcal{O}}_{n}(0)\right\rangle=\frac{n !}{(4 \pi)^{n}\left|x_{1}-x_{2}\right|^{2\left(n+\frac{\lambda}{(24 \pi)^{2}}\right)}}, \quad n ! \sim(2 \pi)^{1 / 2} n^{n+\frac{1}{2}} e^{-n}
$$




\section{Note added}

After this paper appeared, two papers [31, 32] appeared discussing various aspects of the scaling dimension of large charge operators at the WF fixed point in $d=4-\epsilon$. The doublescaling limit explored here involves a different regime than the one explored in these works, which in particular permits a full resummation of Feynmann diagrams to all loop orders. As explained below (3.23), we find the expected agreement for the leading term in the weak coupling expansion.

\section{Acknowledgments}

G.A-T and D.R-G are partially supported by the Spanish government grant MINECO-16FPA2015-63667-P. They also acknowledge support from the Principado de Asturias through the grant FC-GRUPIN-IDI/2018/000174. G.A-T is supported by the Spanish government scholarship MCIU-19-FPU18/02221. J.G.R. acknowledges financial support from projects 2017-SGR-929, MINECO grant FPA2016-76005-C.

\section{A Real space renormalization}

A relevant integral in our discussion is

$$
\mathcal{K}=\frac{1}{G(0, x)^{2}} \int d^{4} z G(0, z)^{2} G(z, x)^{2},
$$

where the propagator is

$$
G(x, y)=\frac{1}{4 \pi^{2}} \frac{1}{(x-y)^{2}} .
$$

After rotation to euclidean signature, the relevant integral to compute is

$$
G(0, x)^{2} \mathcal{K}=-\frac{i}{\left(4 \pi^{2}\right)^{4}} \int d^{4} z \frac{1}{z^{4}(x-z)^{4}} .
$$

The integral can be easily computed following the regularization method of [33], i.e. using that, in $d=4$,

$$
\frac{1}{z^{4}}=-\frac{1}{4} \partial^{2}\left(\frac{\log z^{2} \Lambda^{2}}{z^{2}}\right)
$$

Note that there will be an identical contribution from the divergence at $z=x$, to be regulated just in the same way, and hence the value of the integral will be twice of the contribution at, say $z=0$. We now substitute (A.4) into the integrand of (A.3) and integrate by parts. The resulting integral is convergent upon giving a small imaginary part to $z$, which does not affect the coefficient of the logarithmic term. The integral is then easily computed by going to polar coordinates. One arrives at

$$
\mathcal{K}=-\frac{i}{8 \pi^{2}} \log \left(\Lambda^{2} x^{2}\right)
$$

One may alternatively use the method of [34], which leads to the same result. 
Next, consider the $d=3$ case. The relevant integral is now

$$
\int d^{3} z \frac{1}{z^{3}(x-z)^{3}}
$$

In this case, it can be regularized using the formula

$$
\frac{1}{z^{3}}=-\frac{1}{2} \partial^{2}\left(\frac{\log z^{2} \Lambda^{2}}{z^{2}}\right)
$$

and following just the same steps as in the $d=4$ case.

Open Access. This article is distributed under the terms of the Creative Commons Attribution License (CC-BY 4.0), which permits any use, distribution and reproduction in any medium, provided the original author(s) and source are credited.

\section{References}

[1] S. Hellerman, D. Orlando, S. Reffert and M. Watanabe, On the CFT operator spectrum at large global charge, JHEP 12 (2015) 071 [arXiv:1505.01537] [INSPIRE].

[2] L. Álvarez-Gaumé, O. Loukas, D. Orlando and S. Reffert, Compensating strong coupling with large charge, JHEP 04 (2017) 059 [arXiv:1610.04495] [INSPIRE].

[3] A. Monin, D. Pirtskhalava, R. Rattazzi and F.K. Seibold, Semiclassics, Goldstone bosons and CFT data, JHEP 06 (2017) 011 [arXiv:1611.02912] [inSPIRE].

[4] S. Hellerman, N. Kobayashi, S. Maeda and M. Watanabe, A note on inhomogeneous ground states at large global charge, JHEP 10 (2019) 038 [arXiv:1705.05825] [INSPIRE].

[5] O. Loukas, D. Orlando and S. Reffert, Matrix models at large charge, JHEP 10 (2017) 085 [arXiv: 1707.00710] [INSPIRE].

[6] D. Banerjee, S. Chandrasekharan and D. Orlando, Conformal dimensions via large charge expansion, Phys. Rev. Lett. 120 (2018) 061603 [arXiv:1707.00711] [INSPIRE].

[7] S. Hellerman and S. Maeda, On the large $R$-charge expansion in $\mathcal{N}=2$ superconformal field theories, JHEP 12 (2017) 135 [arXiv:1710.07336] [INSPIRE].

[8] G. Cuomo et al., Rotating superfluids and spinning charged operators in conformal field theory, Phys. Rev. D 97 (2018) 045012 [arXiv:1711. 02108] [INSPIRE].

[9] S. Hellerman et al., Universal correlation functions in rank 1 SCFTs, arXiv:1804.01535 [INSPIRE].

[10] O. Loukas, D. Orlando, S. Reffert and D. Sarkar, An AdS/EFT correspondence at large charge, Nucl. Phys. B 934 (2018) 437 [arXiv:1804.04151] [INSPIRE].

[11] S. Hellerman, N. Kobayashi, S. Maeda and M. Watanabe, Observables in inhomogeneous ground states at large global charge, arXiv:1804.06495 [INSPIRE].

[12] A. De La Fuente, The large charge expansion at large N, JHEP 08 (2018) 041 [arXiv: 1805.00501] [INSPIRE].

[13] S. Favrod, D. Orlando and S. Reffert, The large-charge expansion for Schrödinger systems, JHEP 12 (2018) 052 [arXiv: 1809.06371] [INSPIRE].

[14] D. Banerjee, S. Chandrasekharan, D. Orlando and S. Reffert, Conformal dimensions in the large charge sectors at the O(4) Wilson-Fisher fixed point, Phys. Rev. Lett. 123 (2019) 051603 [arXiv: 1902.09542] [INSPIRE]. 
[15] D. Orlando, S. Reffert and F. Sannino, A safe CFT at large charge, JHEP 08 (2019) 164 [arXiv: 1905.00026] [INSPIRE].

[16] S.M. Kravec and S. Pal, Nonrelativistic conformal field theories in the large charge sector, JHEP 02 (2019) 008 [arXiv: 1809.08188] [INSPIRE].

[17] S.M. Kravec and S. Pal, The spinful large charge sector of non-relativistic CFTs: from phonons to vortex crystals, JHEP 05 (2019) 194 [arXiv:1904.05462] [INSPIRE].

[18] A. Bourget, D. Rodriguez-Gomez and J.G. Russo, A limit for large $R$-charge correlators in $\mathcal{N}=2$ theories, JHEP 05 (2018) 074 [arXiv: 1803.00580] [INSPIRE].

[19] E. Gerchkovitz et al., Correlation functions of Coulomb branch operators, JHEP 01 (2017) 103 [arXiv: 1602.05971] [INSPIRE].

[20] M. Beccaria, On the large $R$-charge $\mathcal{N}=2$ chiral correlators and the Toda equation, JHEP 02 (2019) 009 [arXiv: 1809.06280] [INSPIRE].

[21] M. Beccaria, Double scaling limit of $N=2$ chiral correlators with Maldacena-Wilson loop, JHEP 02 (2019) 095 [arXiv: 1810.10483] [INSPIRE].

[22] A. Grassi, Z. Komargodski and L. Tizzano, Extremal correlators and random matrix theory, arXiv: 1908.10306 [INSPIRE].

[23] R. Rattazzi, G. Badel, G. Cuomo and A. Monin, Semiclass and multi-leg amplitudes, to appear, presented at $24^{\text {th }}$ Recontres Itzykson - Effective field theory in cosmology, gravitation and particle physics, June 5-7, IPhT CEA Saclay, france (2019).

[24] A. Altland and B. Simons, Condensed matter field theory, Cambridge University Press, Cambridge U.K. (2010).

[25] J. Zinn-Justin, Quantum field theory and critical phenomena, International Series of Monographs on Physics, Oxford University Press, U.S.A. (1996).

[26] S. Rychkov and Z.M. Tan, The $\epsilon$-expansion from conformal field theory, J. Phys. A 48 (2015) 29FT01 [arXiv:1505.00963] [INSPIRE].

[27] M.V. Libanov, V.A. Rubakov, D.T. Son and S.V. Troitsky, Exponentiation of multiparticle amplitudes in scalar theories, Phys. Rev. D 50 (1994) 7553 [hep-ph/9407381] [INSPIRE].

[28] M.V. Libanov, D.T. Son and S.V. Troitsky, Exponentiation of multiparticle amplitudes in scalar theories. 2. Universality of the exponent, Phys. Rev. D 52 (1995) 3679 [hep-ph/9503412] [INSPIRE].

[29] D.T. Son, Semiclassical approach for multiparticle production in scalar theories, Nucl. Phys. B 477 (1996) 378 [hep-ph/9505338] [INSPIRE].

[30] https://en.wikipedia.org/wiki/Kermit_the_Frog

[31] G. Badel, G. Cuomo, A. Monin and R. Rattazzi, The $\epsilon$-expansion meets semiclassics, arXiv: 1909.01269 [INSPIRE].

[32] M. Watanabe, Accessing large global charge via the $\epsilon$-expansion, arXiv:1909.01337 [INSPIRE].

[33] D.Z. Freedman, K. Johnson and J.I. Latorre, Differential regularization and renormalization: a new method of calculation in quantum field theory, Nucl. Phys. B 371 (1992) 353 [INSPIRE].

[34] S.M. Chester and S.S. Pufu, Anomalous dimensions of scalar operators in QED 3 , JHEP 08 (2016) 069 [arXiv: 1603.05582] [INSPIRE]. 